\title{
Using Peer Mentors to Screen for CKD at Dialysis Units: Targeting High-Risk Family Members
}

\author{
June E. Swartz, MA; Erica Perry, MSW; Sally Joy, BA; Richard D. Swartz, MD \\ J. Swartz, E. Perry, and S. Joy are with the National Kidney Foundation of Michigan, Ann Arbor, Michigan. Dr. Swartz is with the \\ Division of Nephrology, University of Michigan, Ann Arbor, Michigan.
}

OBJECTIVE: The incidence of chronic kidney disease (CKD) is increasing and affects one in nine individuals in the United States. Genetics and lifestyle factors contribute to the incidence of CKD and serve as screening targets. We screened for predisposition to CKD at dialysis units in southeast Michigan, where obesity and hypertension are common. Families of dialysis patients, as well as non-relatives and staff, were evaluated. Peer Mentors with CKD participated in the screening.

METHODS: "Prevention Fairs" utilized inexpensive screening to determine a predisposition to CKD: urine dipstick for protein, glucose, or blood; BP > 140/90; and body mass index (BMI) > 30. Peer Mentor participation, honoring long-standing patients, and invitations to state legislators were included.

RESULTS: "Fairs" at 16 Michigan centers screened 497 individuals: 61\% (305) had one finding, 18\% (88) had two, and $6 \%(29)$ had three. Obesity was most common (220), and then hypertension (169), proteinuria (41), glycosuria (15), and hematuria (13). Although we had hypothesized that the highest risk would be found among genetic family members, positive screening was not statistically different between genetic relatives and other individuals. In addition, findings were distributed equally across varied demographic settings and races, underscoring the importance of social determinants of health.

DISCUSSION Genetic factors are hypothesized to be predictors for CKD, but these results suggest that CKD risk may also be related to social determinants such as diet, exercise, health consciousness, socioeconomics, or cultural acceptance of obesity. Screening at dialysis centers, enhanced by participation of peers and social activities, contributes to referral for further treatment.

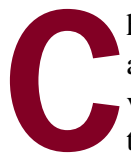
hronic kidney disease (CKD) may affect as many as one in nine individuals in the United States, contributing to the increasing incidence of end-stage renal disease (ESRD) from 258 per million in 1995 to 363 per million in 2006, and to the estimated total ESRD costs for Medicare of $\$ 22.7$ billion in 2006. ${ }^{1}$ Targeting those at risk for CKD in order to detect predisposing characteristics and to refer for early intervention could be important in reducing the incidence and impact of ESRD.

The two most common causes of kidney disease are diabetes mellitus and hypertension, $41 \%$ and $30 \%$, respectively in the State of Michigan. 2,3 Additional studies have linked family history with increased risk of CKD and obesity, ${ }^{4}$ suggesting that screening close relatives of patients with ESRD may be advantageous. ${ }^{5}$ At the same time, African Americans suffer disproportionately from CKD and ESRD, ${ }^{16}$ comprising approximately $14 \%$ of the general population but $45 \%$ of the ESRD population in Michigan, ${ }^{2}$ with poverty among African Americans associated with increased ESRD compared with whites. ${ }^{7}$ Thus, even though genetic relatives of dialysis patients may be more likely to develop kidney disease, ${ }^{8}$ genetics and race alone do not fully explain these predispositions.

The need for earlier diagnosis and increased education is well recognized, ${ }^{6,8,9}$ and the National Kidney Foundation (NKF) has begun to focus increasingly on programs to prevent CKD. Initiated in 2000, the Kidney Early Evaluation Program (KEEP) is a free screening program offered by the NKF to help promote CKD awareness. Eligible participants for KEEP are those adults known to have diabetes, hypertension, or a first-order relative with diabetes, hypertension, or CKD. KEEP performs further evaluation for reduced glomerular filtration rate (GFR; serum creatinine) using a blood test, microalbuminuria, and high blood pressure. KEEP has screened more than 115,000 participants. ${ }^{10,11}$ However, KEEP is relatively expensive (blood draw- ing and urine testing in labs) and has eligibility criteria; thus, more grassroots programs are needed that are less expensive, that detect CKD predisposition among high-risk individuals, and that then refer detected individuals for more intensive evaluation in the KEEP program or medical clinics.

The NKF-Michigan (NKFM) has focused on programs to find high-risk individuals, screen for predisposing factors, and encourage early intervention. As part of this effort, we devised a demonstration project to screen at dialysis units, where family members of ESRD patients were apt to be available for screening. This project utilized volunteers (Peer Mentors from the NKFM) and the easiest, least expensive screening tools (dipstick of urine for protein, blood, or glucose; blood pressure; and weight-height for body mass index [BMI]). The primary targets were genetic relatives of dialysis patients, but screening was extended to willing non-genetic relatives, visitors, and dialysis unit staff as well. 
The format of screening was a "Prevention Fair" that included social activities and invitations to state legislators to attend and present recognition awards for dialysis patients, whose family and friends were invited. Peer Mentors are selected CKD and ESRD patients who undergo 8 hours of formal training to interact with and advise patients. These Peer Mentors encourage individuals both to participate in screening and to seek further referral if they screen positive. Since Peer Mentors are themselves CKD and ESRD patients, they are able to speak first-hand and from direct experience about the importance of further follow up.

A role for Peer Mentors in this project was an important secondary objective for this project. Peer Mentor effectiveness in counseling and education through verbal rather than written communication has been described, specifically using telephone contact in the outpatient management of diabetics, ${ }^{12}$ and using direct contact among ESRD patients to foster understanding and completion of advance directives. ${ }^{13}$ The present project was another opportunity to utilize this important source of trained community volunteer support.

\section{Participants and Methods}

In partnership with several local dialysis providers (William Beaumont Hospitals, University of Michigan Health System, Greenfield Health-Henry Ford Health System, and Fresenius Medical Care), "Prevention Fairs" were held at dialysis units. Medical screening included: 1) urine dipstick for proteinuria $(\geq 2+)$, detectable glycosuria or microhematuria $(\geq 25 \mu \mathrm{L} /$ $\mathrm{mL}$ ); 2) elevated blood pressure (over 140 systolic/90 diastolic) at rest; 3) weight and height measurements to identify "obesity" (BMI $\geq 30$ ); 4) completion of the American Diabetes Association (ADA) diabetes selftest ("Are You At Risk?"); 5) completion of a "Healthy Behavior Checklist" of healthy lifestyle practices to guide Peer Mentors in talking to participants (see below); and 6) Peer Mentors and dialysis nursing staff talking to screened individuals about taking care of themselves and about interacting effectively with the medical system. "Prevention Fairs" were not designed to actually detect CKD by drawing blood for serum creatinine levels; instead, the goal was to prescreen and get any positive participants to early evaluation and treatment.

To increase participation, incentives were offered (e.g., donated tee shirts, pedometers, and water bottles), a raffle was held (prizes including gift certificates and donated hats), and picnics for families were arranged when weather permitted. Fairs were scheduled in each dialysis center on Monday (Mon-Wed-Fri cohort) and Thursday (Tue-Thu-Sat cohort) when this was feasible in order to maximize outreach for as many dialysis families as possible. for presentation of citations and tributes by state legislators to patients with longevity on dialysis. In addition to free medical screening, information was provided about CKD, ESRD-appropriate refreshments, and incentives for healthy behavior. Attendees who did not want medical screening were still asked to fill out the Healthy Behavior Checklist and the ADA self-test for diabetes.

Data collected were analyzed statistically using the standard Chi-square test for the frequency of findings among the several groups under consideration.

Healthy behavior checklist for people at risk for chronic kidney disease

- I want to stay healthy to prevent kidney disease.

- I do not smoke or have quit smoking.

- I eat five or more fruits and vegetables each day.

- I exercise at least 20 minutes, three times per week (walking counts).

- I avoid drinks such as soda that are loaded with sugar.

- I want my relatives to get/stay healthy.

- I encourage my relatives to have annual doctor appointments.

- A relative I encouraged will see or already saw a doctor this month.

Peer Mentors were available to talk with all screened individuals, focusing attention on free follow-up programs through NKF-M that include:

- A 6-week chronic disease selfmanagement program known as Personal Action Toward Health (PATH), an evidence-based program that is designed to benefit both adults with long-term health problems (such as diabetes, CKD, and heart disease) and family members and caregivers ${ }^{14}$

- Enhance Fitness, a three-times-a-week physical activity program for combating obesity, led by a certified fitness instructor and providing social interaction, cardiovascular conditioning, and strength, flexibility, and balance training

- The Kidney Early Evaluation Program (KEEP) from NKF for those already identified though primary screening and at highest risk for progressive CKD and ESRD, for more intensive testing and treatment referral.

The "Prevention Fairs" also focused on dialysis patients themselves, arranging
This study was reviewed and approved by the Investigational Review Board of the Michigan Department of Community Health, with all participants signing consent forms.

\section{Results}

"Prevention Fairs" were held on one or two days at 16 distinct dialysis centers in the Detroit metropolitan area of southeast Michigan, beginning with Beaumont, Royal Oak, in 2007. The characteristics of the dialysis centers and the individuals screened at each center are summarized in Table I. Overall, more than 520 individuals participated directly in the activities at the centers, and 497 (95\% of participants) were screened for CKD risk factors. A mix of urban and suburban centers was included, with dialysis census ranging from 37 to 270 patients. The number of individuals screened at each center varied roughly with the size of the center but was also influenced by the degree of enthusiasm and participation among the staff. African Americans comprised 13-96\% of 


\section{Screening for CKD}

\begin{tabular}{|c|c|c|c|c|c|c|}
\hline $\begin{array}{l}\text { Facility } \\
\text { (no. of “Fairs") }\end{array}$ & $\begin{array}{l}\text { Facility } \\
\text { Size }\end{array}$ & $\begin{array}{l}\text { Persons } \\
\text { Screened }\end{array}$ & Female & $\begin{array}{l}\text { African- } \\
\text { American }\end{array}$ & $\begin{array}{c}\text { Genetic } \\
\text { Relatives }\end{array}$ & Age $^{*}$ \\
\hline & $n$ & $n$ & $n(\%)$ & $n(\%)$ & $n(\%)$ & $\mathrm{Y} \pm \mathrm{SD}$ \\
\hline \multicolumn{7}{|l|}{ William Beaumont } \\
\hline Royal Oak (1) & 159 & 8 & $6(75)$ & 1 (13) & $2(25)$ & $61 \pm 15$ \\
\hline Hazel Park (1) & 104 & 15 & $9(60)$ & 7 (47) & $6(40)$ & $44 \pm 12$ \\
\hline Troy (2) & 123 & 40 & $29(74)$ & $6(15)$ & $10(26)$ & $54 \pm 15$ \\
\hline \multicolumn{7}{|l|}{ University of Michigan } \\
\hline Livonia (2) & 90 & 33 & $23(79)$ & $23(79)$ & $20(68)$ & $45 \pm 15$ \\
\hline Ann Arbor (2) & 120 & 38 & $24(65)$ & $13(35)$ & $12(32)$ & $49 \pm 17$ \\
\hline \multicolumn{7}{|l|}{ Greenfield } \\
\hline Taylor (2) & 120 & 59 & $33(58)$ & $22(39)$ & $22(41)$ & $49 \pm 17$ \\
\hline W Pavilion (1) & 270 & 46 & $35(78)$ & $43(96)$ & $30(67)$ & $41 \pm 15$ \\
\hline Southfield (1) & 180 & 23 & $13(54)$ & $22(92)$ & $12(50)$ & $38 \pm 15$ \\
\hline Fairlane (2) & 210 & 43 & $28(57)$ & $26(53)$ & $24(52)$ & $50 \pm 17$ \\
\hline NW Dialysis (2) & 260 & 50 & $31(63)$ & $43(88)$ & $13(27)$ & $44 \pm 14$ \\
\hline St. Marys (2) & 75 & 36 & $23(64)$ & $26(72)$ & $19(53)$ & $46 \pm 17$ \\
\hline \multicolumn{7}{|l|}{ Fresenius } \\
\hline Romulus (2) & 37 & 20 & $14(64)$ & $6(27)$ & $10(46)$ & $44 \pm 16$ \\
\hline University of Detroit (2) & 138 & 22 & $13(57)$ & 22 (95) & $10(44)$ & $41 \pm 14$ \\
\hline Bewick (1) & 71 & 16 & $9(56)$ & $12(75)$ & $5(32)$ & $38 \pm 14$ \\
\hline Botsford (1) & 127 & 18 & $13(72)$ & $8(44)$ & $10(56)$ & $44 \pm 16$ \\
\hline Livonia (2) & 119 & 30 & $19(63)$ & $11(37)$ & $12(40)$ & $48 \pm 14$ \\
\hline Total (26) & & 497 & $321(65)$ & $291(59)$ & 217 (44) & $46 \pm 16$ \\
\hline
\end{tabular}

screened individuals depending on location and referral patterns; females were in the majority at all centers because of the predominance of women among staff members screened. (Visitors and staff members were all asked whether they had a relative on dialysis.) Among screened individuals, genetic relatives comprised $44 \%$, ranging from 25 to $67 \%$ among the dialysis units, and age was generally in the 5th decade, with minor variations that depended on each unit's demographics and did not vary except for a single unit with small numbers and older patients.

Table II shows the results of screening for CKD predilection, comparing the 217 genetic relatives and 280 other individu- als (non-genetic relatives, visitors, and dialysis staff). All "non-genetic" relatives, visitors, and staff were questioned as to whether they had a relative who was on dialysis or had CKD; if so, they were then included in the "genetic" group. Forty-one percent (89) of screened genetic relatives and $35 \%$ (99) of other individuals had one abnormality, $18 \%$ (40) of genetic relatives and $17 \%$ (48) of others had two abnormalities, and $8 \%$ (17) of genetic relatives and $4 \%$ (12) of others had three abnormalities. Thus, more than $61 \%$ (305) of all screened individuals had at least one abnormality, and positive screening was slightly higher among genetic relatives but was not statistically significant ( $p=0.064$, Chi-square test). Positive screening was not higher among African Americans compared with other races ( $p=0.237$, Chi square), and the distribution of abnormalities did not vary statistically in a comparison of the different centers where the "Prevention Fairs" were conducted ( $p=0.351$, Chi square) despite the geographical and environmental differences.

Among the abnormal findings, 220 were "obesity," 169 were "hypertension," 41 were proteinuria, 18 were glycosuria, and 14 were hematuria (excluding menstruating women). Participants with two or more abnormalities $(n=117)$ received follow-up telephone calls; 95/117 (81\%) were reached, and $60 / 117(51 \%)$ said that they 
TABLE II. Results of screening at each dialysis facility. ${ }^{*}$

\begin{tabular}{|c|c|c|c|c|c|c|c|c|c|c|}
\hline & \begin{tabular}{|c|} 
Genetic \\
Relatives \\
Screened \\
\end{tabular} & $\begin{array}{l}1 \text { Abnl } \\
\text { Result }\end{array}$ & $\begin{array}{l}2 \text { Abnl } \\
\text { Results }\end{array}$ & $\begin{array}{r}3 \text { Abnl } \\
\text { Results }\end{array}$ & $\begin{array}{l}\text { Total } \\
\text { Abnl }\end{array}$ & $\begin{array}{c}\text { Other } \\
\text { Individuals } \\
\text { Screened }\end{array}$ & $\begin{array}{l}1 \text { Abnl } \\
\text { Result }\end{array}$ & $\begin{array}{l}2 \text { Abnl } \\
\text { Results }\end{array}$ & $\begin{array}{r}3 \mathrm{Abnl} \\
\text { Results }\end{array}$ & $\begin{array}{l}\text { Total } \\
\text { Abnl }\end{array}$ \\
\hline \multicolumn{11}{|l|}{ Beaumont Dialysis } \\
\hline Royal Oak & 2 & 2 & 0 & 0 & 2 & 6 & 3 & 1 & 0 & 4 \\
\hline Hazel Park & 6 & 3 & 1 & 0 & 4 & 9 & 3 & 0 & 0 & 3 \\
\hline Troy & 10 & 2 & 1 & 3 & 6 & 30 & 14 & 1 & 1 & 16 \\
\hline \multicolumn{11}{|l|}{ University of Michigan } \\
\hline Livonia & 20 & 9 & 3 & 1 & 13 & 13 & 4 & 4 & 1 & 9 \\
\hline Ann Arbor & 12 & 4 & 4 & 1 & 9 & 26 & 8 & 4 & 2 & 14 \\
\hline \multicolumn{11}{|c|}{ Greenfield Health System } \\
\hline Taylor & 22 & 6 & 3 & 5 & 14 & 37 & 14 & 5 & 4 & 23 \\
\hline Pavilion & 30 & 16 & 1 & 0 & 17 & 16 & 3 & 3 & 1 & 7 \\
\hline NW Dialysis & 12 & 6 & 3 & 1 & 10 & 11 & 3 & 3 & 0 & 6 \\
\hline Fairlane & 24 & 7 & 4 & 4 & 15 & 19 & 6 & 6 & 0 & 12 \\
\hline Northwest & 13 & 9 & 4 & 0 & 13 & 37 & 16 & 2 & 1 & 19 \\
\hline St. Mary's & 19 & 5 & 7 & 1 & 13 & 17 & 5 & 6 & 0 & 11 \\
\hline \multicolumn{11}{|l|}{ Fresenius } \\
\hline Romulus & 10 & 3 & 2 & 0 & 5 & 10 & 6 & 1 & 0 & 7 \\
\hline University of Detroit & 10 & 5 & 3 & 0 & 8 & 12 & 6 & 2 & 0 & 8 \\
\hline Berwick & 5 & 3 & 0 & 0 & 3 & 11 & 0 & 3 & 1 & 4 \\
\hline Botsford & 10 & 4 & 1 & 1 & 6 & 8 & 4 & 1 & 0 & 5 \\
\hline Livonia & 12 & 5 & 3 & 0 & 8 & 18 & 4 & 6 & 1 & 11 \\
\hline Total screened & 217 & 89 & 40 & 17 & 146 & 280 & 99 & 48 & 12 & 159 \\
\hline$\%$ of Total & & 41 & 18 & 8 & 67 & & 35 & 17 & 4 & 57 \\
\hline
\end{tabular}

had consulted with a physician for further evaluation and treatment.

The ADA self-test was completed by 492 individuals, and letters to those scoring "high risk" were mailed to 188 individuals. An additional 332 individuals completed the informal Healthy Behavior Checklist. With the advent of new programs during the second year of the project, referrals were made for 18 individuals who agreed to attend the NKFM PATH healthy eating nutrition program; 35 obese individuals were referred to Enhance Fitness, and 30 persons were referred to KEEP for more specific CKD evaluation. In all, 78 Peer Mentors participated, and 17 different state legislators attended the "Prevention Fairs" and were updated about CKD, ESRD, and preventive programs.

\section{Discussion}

The present demonstration project was originally aimed at screening for CKD predisposition among predictably high-risk individuals, namely, family members of dialysis patients. By conducting screening at the dialysis center itself, we increased access to these individuals but also had an opportunity to offer screening to other visitors and staff. Although we hypothesized that genetic family members would have the highest prevalence of screening abnormalities, ${ }^{15}$ our screening results suggest that the social determinants of health, such as socioeconomic status and social and environmental stressors, may have a "non-genetic" role in the predilection to CKD. These findings are similar to those articulated in two other interesting studies, first, the Framingham report concerning obesity by Christakis et al., in which obesity appears to cluster in social groups independent of genetic and family relationships ${ }^{16}$; and second, the report by Tsai et al. from Taiwan reporting the prevalence of CKD among spouses of dialysis patients. ${ }^{17}$

Our screening program detected at least one recognized risk factor in more than $61 \%$ of 497 screened individuals, considerably 


\section{Screening for CKD}

more than expected and more than has been reported in other settings. The most frequent risk factor in our survey was obesity, supporting previous reports showing that obesity is a strong and independent predictor of CKD with microalbuminuria. ${ }^{4}$ In our original proposal, we also hypothesized that African Americans would have proportionally more abnormalities; however, our results showed that the frequency of positive screening undertaken in the dialysis setting did not differ between urban and suburban sites that had varying racial and social composition. Our results suggest that in the dialysis units where "Prevention Fairs" were conducted, even the staff, many of whom also lived in economically depressed areas, appeared to be subject to environmental exposure that might affect their health, including diet, stress, or acceptance of unhealthy behavior as the norm. ${ }^{6}$

Both the actual frequency of positive screening and the clustering on the basis of factors other than heredity underscore the need to get the attention of screened individuals in order to initiate preventive referral and further care. Previous findings, particularly those of the 2004 National Health and Nutrition Examination Survey (NHANES), found that $32.2 \%$ of U.S. adults met clinical criteria for obesity, lend urgency to this endeavor. ${ }^{18}$ In Michigan, the 2007 Michigan Behavioral Risk Factor Surveillance Survey (BRFSS) reported that the prevalence of obesity was $28.4 \%$ (BMI > 30) and that only $22 \%$ of adults eat USDA-recommended servings of fruits and vegetables, while only $50 \%$ engage in the recommended physical activity. ${ }^{19}$ Compounding these findings is the frequent difficulty that many high-risk adults have in accessing physical training or nutritional education resources. Since lifestyle modification appears to modulate the incidence of diabetes and $\mathrm{CKD},{ }^{20}$ the importance of the present demonstration project is not simply in the screening itself but in focusing on high-risk individuals and getting them motivated to change and visit a clinic.

This methodology in this project has several important advantages. The screening procedures utilized were basic, inexpensive, and easy to administer; the project included volunteer Peer Mentors; and the project fostered partnership between NKF-M and the dialysis unit staff, who knew the patients and families and were in the best position to encourage attendance and participation for screening within the community. Other CKD screening programs such as KEEP are more expensive, utilizing blood drawing and outside laboratory testing, and these programs access individuals who meet criteria that include known diabetes, hypertension, or a first-order relative with diabetes, hypertension, or CKD. ${ }^{21}$ In addition, follow-up on KEEP participants was by questionnaire forms mailed to them, and the response rate was $28.4 \%,{ }^{10}$ whereas the present demonstration project telephoned those with two or more abnormalities $(n=117)$, reaching $81 \%$ $(n=95)$ of whom more than $50 \%$ consulted a medical caregiver. Many were referred to free medical clinics.

Important additional results of this demonstration project, over and above the actual screening itself, are several: establishing an atmosphere in which Peer Mentors can encourage individuals to participate in screening; providing in-depth education regarding the stress to caregivers and the pathway lying ahead for CKD and ESRD; making the secondary referrals for fitness coaching, nutrition, and further medical evaluation and treatment; and developing a network of experienced individuals who can help with understanding and dealing with the medical system within the community. Thus, screening was only the first step; opening the door to further follow-up by utilizing the social networking available through the dialysis unit and the NKF-M was an important second step. In addition, the recognitions and tributes that were given in ceremonies that included state legislators and dialysis unit administrators put a "positive face" on the entire dialysis setting that is unusual in the present economic and healthcare environment.

Several difficulties and shortcomings were encountered during this demonstration project. For all of their personal and psychosocial impact in this type of outreach, reliance on volunteer Peer Mentors leaves the ongoing schedule subject to the difficulties that sometimes befall Peer Mentors, who are themselves dialysis or transplant patients. Despite these difficulties, Peer Mentors were invaluable in promoting trust and credibility, especially among various ethnic populations like African Americans. Another difficulty was publicizing our events, especially in urban units in which family members did not ordinarily accompany patients, often limiting the number of persons available for screening; our total number screened was under 500. Even so, the consistency observed across units with varying demographic make-up and geographic environment was striking and suggests that our findings are applicable more generally. D\&T

\section{References}

1. US Renal Data System: USRDS 2008 Annual Data Report. Bethesda, MD: The National Institutes of Health, National Institute of Diabetes and Digestive and Kidney Diseases; 2008.

2. ESRD Annual Data Report, Renal Network 11; 2008.

3. Bash L, Astor B, Coresh J. Risk of incident ESRD: a comprehensive look at cardiovascular risk in factors and 17 years of follow-up in the Atherosclerosis Risk in Communities (ARIC) Study. Am J Kidney Dis. 2010;55:31-41.

4. Bello A, Peters J, Wright J, De Zeeuw D, El Nahas M. A population-based screening for microalbumin uria among relatives of CKD patients: the Kidney Evaluation and Awareness Program in Sheffield (KEAPS). Am J Kidney Dis. 2008;52:434-443.

5. Freedman B, Volkova N, Satko S, et al. Populationbased screening for family history of end-stage renal disease among incident dialysis patients. Am J Nephrol. 2005;25:529-535.

6. Waterman A, Browne T, Waterman B, Gladstone E, Hostetter T. Attitudes and behaviors of African Americans regarding early detection of kidney disease. Am J Kidney Dis. 2008;51:554-562.

7. Volkova N, McClellan W, Klein M, et al. Neighborhood poverty and racial differences in ESRD incidence. Am J Kidney Dis. 2008;19:356-364.

8. McClellan W, Satko S, Gladstone E, Krisher J, Narva A, Freedman B. Individuals with a family history of ESRD are a high-risk population for CKD: implications for targeted surveillance and intervention activities. Am J Kidney Dis. 2009;53. S100-S106.

9. Flessner M, Wyatt S, Akylbekova E, et al. Prevalence and awareness of CKD among African Americans: the Jackson Heart Study. Am J Kidney Dis. 2009;53:238-247.

10. Collins A, Li S, Chen S, Vassalotti J. Participant follow-up in the Kidney Early Evaluation Program (KEEP) after initial detection. Am J Kidney Dis. 2008;51:S69-S76.

11. Whaley-Connell A, Sowers J, McCullough P, et al. Diabetes mellitus and CKD awareness: the Kidney Early Evaluation Program (KEEP) and National Health and Nutrition Examination Survey (NHANES). Am J Kidney Dis. 2009;53:S11 S21.

12. Heisler M, Piette J. Ihelpyou, and you help me: facilitated telephone peer support among patients with diabetes. Diabetes Educ. 2005;31:869-879

13. Perry E, Swartz J, Brown S, Smith D, Kelly G, Swartz R. Peer mentoring: a culturally sensitive approach to end-of-life planning for long-term dialysis patients. Am J Kidney Dis. 2005;46:111119.

14. Gordon C, Galloway T. Review of findings on Chronic Disease Self-Management Program (CDSMP) outcomes: physical, emotional \& health-related quality of life, healthcare utilization and costs. Atlanta, 
GA: Centers for Disease Control and Prevention, National Council on Aging; 2008.

15. Metzler M. Social determinants of health: what, how, why, and now. Prev Chronic Dis. 2007:4: A85.

16. Christakis N, Fowler J. The spread of obesity in a large social network over 32 years. N Engl J Med. 2007;357:370-379.

17. Tsai J, Chen S, Hwang S, Chang J, Lin M, Chen H. Prevalence of risk factors for CKD in spouses and relatives of hemodialysis patients. Am J Kidney Dis. 2010;55:856-866.
18. Bomback A, Kshirsagar A, Whaley-Connell A. et al. Racial differences in kidney function among individuals with obesity and metabolic syndrome: results from the Kidney Early Evaluation Program (KEEP). Am J Kidney Dis. 2010;55: S4-14.

19. Michigan BRFSS Surveillance Brief. Available at: http: // www.Michigan.gov/documents / MDCH/MIBRFSS_surveillance_brief; _June 2008.

20. Levey A, Schoolwerth A, Burrows N, Williams D, Rabon Stith K, McClellan W. Comprehensive public health strategies for preventing the development, progression, and complications of CKD: report of an expert panel convened by the Centers

for Disease Control and Prevention. Am J Kidney Dis. 2009;53:522-535.

21. McCullough P, Li S, Jurkowitz C, et al. CKD and cardiovascular disease in screened high-risk volunteer and general populations: the early Kidney and Evaluation Program (KEEP) and National Health and Nutrition Examination Survey (NHANES) 1999-2004. Am J Kidney Dis. 2008;51(suppl 2):s38-s45. 